\title{
Potential of Recent Ambient lonization Techniques for Future Food Contaminant Analysis Using (Trans)Portable Mass Spectrometry
}

\author{
Marco H. Blokland ${ }^{1}$ (I) $\cdot$ Arjen Gerssen ${ }^{1} \cdot$ Paul W. Zoontjes ${ }^{1} \cdot$ Janusz Pawliszyn ${ }^{2} \cdot$ Michel W.F. Nielen $^{1,3} \mathbb{C}$
}

Received: 3 June 2019 / Accepted: 31 October 2019 / Published online: 16 December 2019

(C) The Author(s) 2019

\begin{abstract}
In food analysis, a trend towards on-site testing of quality and safety parameters is emerging. So far, on-site testing has been mainly explored by miniaturized optical spectroscopy and ligand-binding assay approaches such as lateral flow immunoassays and biosensors. However, for the analysis of multiple parameters at regulatory levels, mass spectrometry (MS) is the method of choice in food testing laboratories. Thanks to recent developments in ambient ionization and upcoming miniaturization of mass analyzers, (trans)portable mass spectrometry may be added to the toolkit for on-site testing and eventually compete with multiplex immunoassays in mixture analysis. In this study, we preliminary evaluated a selection of recent ambient ionization techniques for their potential in simplified testing of selected food contaminants such as pesticides, veterinary drugs, and natural toxins, aiming for a minimum in sample preparation while maintaining acceptable sensitivity and robustness. Matrix-assisted inlet ionization (MAI), handheld desorption atmospheric pressure chemical ionization (DAPCI), transmission-mode direct analysis in real time (TM-DART), and coated blade spray (CBS) were coupled to both benchtop Orbitrap and compact quadrupole single-stage mass analyzers, while CBS was also briefly studied on a benchtop triple-quadrupole MS. From the results, it can be concluded that for solid and liquid sample transmission configurations provide the highest sensitivity while upon addition of a stationary phase, such as in CBS, even low $\mu \mathrm{g} / \mathrm{L}$ levels in urine samples can be achieved provided the additional selectivity of tandem mass spectrometry is exploited.
\end{abstract}

Keywords Food analysis $\cdot$ Food contaminants $\cdot$ Mass spectrometry $\cdot$ Ambient ionization $\cdot$ On-site testing

\section{Introduction}

In food quality and safety control, traditional workflows comprise on-site sampling by food inspectors from authorities or industry, administration and transportation of the samples to centralized laboratories, pre-screening of the samples for target substances followed by confirmatory analysis in case of suspect results, and finally, reporting and appropriate followup action in case of non-compliant samples. On the one hand, one may argue that this control approach has been effective:

Michel W.F. Nielen

michel.nielen@wur.nl

1 Wageningen Food Safety Research (WFSR), part of Wageningen University \& Research, P.O. Box 230, 6700

AE Wageningen, The Netherlands

2 Department of Chemistry, University of Waterloo, 200 University Avenue West, Waterloo, Ontario N2L3G1, Canada

3 Laboratory of Organic Chemistry, Wageningen University, Stippeneng 4, 6708 WE Wageningen, The Netherlands food has never been safer in history. On the other hand, the efficacy and cost-efficiency of this approach might be questioned: millions of compliant samples are unnecessarily transported and analyzed while food scandals and food fraud still frequently occur. A trend towards on-site pre-screening of food quality and safety parameters can be observed. Portable and smartphone-based optical spectroscopy instruments such as near-infrared (NIR) scanners and hyperspectral cameras are already on the market for the analysis of the macrocomposition in foods and drinks, including adulterations thereof (Liu et al. 2018; Rateni et al. 2017). Specific substances such as antibiotics residues are already analyzed in milk tanks at the farm by dairy truck drivers using ligandbinding lateral flow assays and possibly soon consumers will test food themselves (Ross et al. 2018). It seems rather unlikely that the optical food scanners will be capable of analyzing food contaminants at the regulated levels which are typically at $\mu \mathrm{g} / \mathrm{L}$ or $\mu \mathrm{g} / \mathrm{kg}$ concentration. Despite the possibility of multiplexing, on-site application of ligand-binding assays will be restricted by the scope of the available biorecognition elements. Although not established yet in food quality and safety 
control laboratories, it is envisaged that recent academic developments in portable mass spectrometry (MS) (Snyder et al. 2016; Pulliam et al. 2015; Zhai et al. 2017; Brkic et al. 2018) will find its way to commercial products with simplified tablet or smartphone-based user interfaces which eventually will lead to on-site food contaminant analysis. A prerequisite for such a development is the availability of simplified sample preparation and sample introduction protocols. In the last decade, ambient ionization techniques have been developed that offer such simplicity, at least in an academic laboratory environment. Dozens of ambient ionization approaches have been proposed and discussed in a large number of review papers (Venter et al. 2008; Harris et al. 2011; Smoluch et al. 2016; Lu et al. 2018; Feider et al. 2019). Following the introduction of droplet impact-based techniques, such as desorption electrospray ionization (DESI) (Takáts et al. 2004; Cooks et al. 2006); plasma-based techniques, such as direct analysis in real time (DART) (Cody et al. 2005) and low temperature plasma (LTP) (Harper et al. 2008); laser-based desorption techniques, such as laser ablation electrospray ionization (LAESI) (Nemes and Vertes 2007); and other techniques such as paper spray (PS) (Liu et al. 2010), rapid evaporative ionization mass spectrometry (REIMS) (Schäfer et al. 2009), and atmospheric pressure solids analysis probe (ASAP) (McEwen et al. 2005) have been developed. With respect to the potential of these techniques for the ionization of selected food contaminants, several initial and encouraging examples can be found in literature (Schurek et al. 2008; Garcia-Reyes et al. 2009; Hajslova et al. 2011; Nielen et al. 2011; Farré et al. 2013; Nielen and van Beek 2014; Lu et al. 2018), but hardly any studies evaluated ambient ionization of food contaminants having the requirements of (trans)portable MS in mind (Ma et al. 2016; Pulliam et al. 2015; Soparawalla et al. 2011; Huang et al. 2010). Aiming for future coupling with portable mass spectrometry, some practical and analytical requirements for simplified ambient ionization techniques can be summarized as follows: (i) no vulnerable laser set-ups, (ii) no heavy gas cylinders, (iii) small footprint, (iv) low weight, (v) low power consumption, (vi) robustness, and (vii) acceptable analytical performance for pre-screening performance, i.e., a low risk of false-negative results. Based on these considerations, a few recent ambient ionization techniques were selected for their potential in simplified testing of food contaminants.

An ultimate simplified ionization does not require gases, high voltages, or vulnerable lasers. Matrix-assisted inlet ionization (MAII) was developed in the Trimpin group ( $\mathrm{Li}$ et al. 2012; Trimpin and Inutan 2013) and even demonstrated on a compact single-quadrupole MS (Devereaux et al. 2016). In MAI, the sample is mixed with a matrix such as 3nitrobenzonitrile or 2,5-dihydroxybenzoic acid and simply tapped against the atmospheric inlet (heated capillary or sample cone) of the MS. The ionization mechanism seems to be rather magic and is still under debate (Trimpin 2016) but encouraging results have been presented, although not much yet beyond the inventor's laboratory. Our second simplified approach is based on a prototype handheld desorption atmospheric pressure chemical ionization (DAPCI) probe. DAPCI was introduced in the early days of ambient ionization (Chen et al. 2007) but not much applied yet to food contaminants. Recently, a prototype battery-operated handheld DAPCI gun has been developed that simply aspirates ambient air with a built-in miniaturized membrane pump (Jjunju et al. 2015). As a third approach, we evaluated transmission-mode direct analysis in real time (TM-DART) (Harding et al. 2014). Following the initial DART developments at Jeol Inc. (Cody et al. 2005), a transmission-mode version (ID-Cube ${ }^{\mathrm{TM}}$ ) was developed at IonSense Inc. in which the sample is spotted on a metal grid fitted in cardboard card. Upon resistive heating, the sample is desorbed and plasma ionized. Currently, this compact device requires a supply of helium gas, but it has been shown already that DART can successfully operate with ambient air as well, although at the cost of sensitivity (Harris et al. 2015). As a fourth approach, we briefly evaluated coated blade spray (CBS). CBS has been recently developed by the Pawliszyn group (Gómez-Ríos and Pawliszyn 2014; Gómez-Ríos et al. $2017 \mathrm{a}, \mathrm{b}$ ) and is considered highly promising since it combines solid-phase microextraction (SPME) (Souza-Silva et al. 2015) onto a conductive metal strip, featuring both (selective) sample enrichment and direct spray into a MS by applying a high voltage onto the strip.

In this study, we preliminary evaluated these recent simplified ambient ionization techniques for their potential in the testing of selected food contaminants such as pesticides, veterinary drugs, and natural toxins, aiming for a minimum in sample preparation while maintaining acceptable sensitivity and robustness. As outlined above, due to the still limited commercial availability of truly portable MS equipment, we performed our evaluation using benchtop instruments in the lab: a single-stage Orbitrap, a triple-quadrupole tandem MS, and a transportable $(35 \mathrm{~kg}$ ) compact single-quadrupole MS system (Bu et al. 2016). Eventually, based on theoretical selectivity considerations (Berendsen et al. 2013; Berendsen et al. 2015), we envisage that a portable tandem MS mass analyzer, either ion trap or triple quadrupole (Wright et al. 2015) will be the minimum requirement for future on-site testing of food contaminants at regulatory levels.

\section{Materials and Methods}

\section{Chemicals}

Metolachlor, chlormequat, atrazine, and imazalil were obtained from LGC standards (Wessel, Germany); clenbuterol, salbutamol, salmeterol, formoterol, and higenamine were obtained from Witega (Berlin, Germany); zilpaterol was kindly 
provided by EURL (Berlin, Germany); stanozolol was obtained from NMI (Sydney, Australia); cortisol was obtained from Steraloids (Newport, RI, USA); levofloxacin, tetracycline dapsone, and 3-nitrobenzonitrile (3-NBN) were obtained from Sigma-Aldrich (St. Louis, MO, USA); domoic acid was obtained from Tocris Bioscience (UK); and paracetamol(-D4), ibuprofen, diclofenac, colchicine, and terfenadine were a kind gift of the British Mass Spectrometry Society. Acetonitrile $(\mathrm{ACN})$, acetic acid, formic acid (FA), and sodium hydroxide were obtained from Actu-All (Oss, The Netherlands). Water was purified using a Milli-Q system from Millipore (Bedford, MA, USA). Paracetamol and diclofenac pills were obtained from a local drugstore; lemons, blueberries, pumpkin, and apple were purchased from a local supermarket. Helium gas (purity > 5.0) was used for the transmission DART experiments.

\section{Instruments and Consumables}

Microscope slides were obtained from Thermo Scientific (Waltman, MA, USA). A high voltage power supply (model PS350/5000 V-25 W) was obtained from Stanford Research Systems, Inc. (Sunnyvale, CA, USA). ID-CUBE, ID-CUBE current regulator model ID-100, OpenSpot sample cards, DIPit Samplers, and the DART-SVP ion source were obtained from IonSense (Saugus, MA, USA). A custom-designed handheld DAPCI ion source was purchased from QTechnologies (Liverpool, UK). The blades used for CBS were developed and coated with Agilent bondelut certify stationary phase at Waterloo University (Waterloo, ON, Canada). The mass spectrometers used were a Thermo Scientific model Exactive benchtop Orbitrap MS (Thermo Fisher Scientific, San Jose, CA, USA), a Waters (Milford, MA, USA) ACQUITY QDa MS detector, a Waters quadrupole Time-offlight MS (Xevo-QTOF) and a Waters Quattro Ultima triplequadrupole tandem MS system.

\section{MAll Measurements}

The QTOF ion source was modified as such that voltages and gasses remained but the sample cone was directly accessible for MAII experiments. The modification was done by removing the protective front window of a nano-ESI source (Waters). For the MAII experiments, the capillary voltage and gas flow were switched off, the sample cone was set at $10 \mathrm{~V}$, extraction cone $1 \mathrm{~V}$, and the source temperatures were $150{ }^{\circ} \mathrm{C}$. 3-NBN matrix was prepared by dissolving $5 \mathrm{mg}$ in $100 \mu \mathrm{L}$ of $\mathrm{ACN}$ :water $(1: 1 \mathrm{v} / \mathrm{v} \%)$. From a given matrix:analyte mixture, $1 \mu \mathrm{L}$ was typically spotted on the target slide, the spot was allowed to dry before gently tapping against the sample cone. Solutions of the analytes (1 ppm) were prepared in ACN:water $(1: 1 \mathrm{v} / \mathrm{v})$ with $0.1 \% \mathrm{FA}$. The blueberry was spiked with $5 \mathrm{ng}$ chlormequat and left to dry for $20 \mathrm{~min}$. Each sample spot was tapped for $60 \mathrm{~s}$; after measurement, the ion abundance was averaged by XCalibur software (version 4.1) and the average intensity of the recorded mass of interest was used for further calculations.

\section{DAPCI Measurements}

To perform DAPCI analysis, both the Exactive Orbitrap and QDa source were modified. The front of the Orbitrap source was opened, and the security lock was overruled by placing a small pin in the lock. The QDa was modified by removing the ESI source housing, attaching a curved adapter onto the skimmer cone and changing the instrument setting according to Trimpin et al. (Devereaux et al. 2016). The following settings were applied for the Orbitrap: ion-source voltage $3 \mathrm{kV}$, tube lens $200 \mathrm{~V}$, heated capillary voltage $25 \mathrm{~V}$, heated capillary temperature $250{ }^{\circ} \mathrm{C}$, maximum injection time $50 \mathrm{~ms}$, micro scans 1 . The MS data were recorded as profile spectra for an average time of 30-60 s. Spectral quality was improved by background spectra subtraction using the Xcalibur software (version 4.1). For the QDa experiment, the following settings were used, gas flow 2 a.u., cone voltage $15 \mathrm{~V}$, and data acquisition rate was set at $67 \mathrm{~Hz}$; all experiments were performed in positive ion mode.

For DAPCI Orbitrap experiments, a few drops of the sample extracts were transferred to a microscope slides and subsequently air-dried for approximately $3 \mathrm{~min}$ before MS analysis. The microscope slide is then clamped and placed between the outlet of the DAPCI source and the inlet of the mass spectrometer. The angle between the DAPCI and the sample was kept at approximately $30^{\circ}$ and the angle between the clamped microscope slide and the MS at approximately $20^{\circ}$. For DAPCI QDa experiments, samples were transferred onto a Dip-It sampler and air-dried for approximately $3 \mathrm{~min}$ before analysis. The distance between both the Dip-It sampler and the MS, as well as the sampler and the DAPCI device, was approximately $10 \mathrm{~mm}$.

\section{Transmission-DART Measurements}

For TM-DART, both the Orbitrap and QDa source were modified. The Orbitrap ESI source was removed to place the TMDART source in front of the heated inlet capillary. Furthermore, an adapter containing a resistor was inserted to overrule security locks and mimic the presence of an ESI source for the software. Comparable as with the MAII experiments a small pin was placed in such a way that the interlock was overruled. The modifications for the QDa were comparable as described for the DAPCI experiments. Similar MS settings as the DAPCI were used for the TM-DART experiments. In the TM-DART experiment, the current regulator was set on high (ca. $450{ }^{\circ} \mathrm{C}$ ), the helium flow rate set at $2 \mathrm{~L} / \mathrm{min}$ and the ionization energy at $1 \mathrm{kV}$. For the DART-SVP experiment, 
settings were grid voltage $350 \mathrm{~V}$, temperature setting $350{ }^{\circ} \mathrm{C}$, helium gas flow rate 75-80 psi, and the open-air gap distance was maintained at approx. $1.0 \mathrm{~cm}$. For the TM-DART-QDa measurements, the DART ion source was placed in front of the curved tube at approx. $0.6 \mathrm{~cm}$ open-air gap distance. For TMDART measurements, aliquots of $5 \mu \mathrm{L}$ were pipetted onto the stainless steel mesh of OpenSpot sample cards and were dried by gently applying a nitrogen flow. The sample card was then placed in the slot of the TM-DART. After a few seconds, the current regulator was turned on which resulted in a short temperature program for $30 \mathrm{~s}$. The helium flow rate was set to 3 , and the temperature was set to high $\left(450^{\circ} \mathrm{C}\right)$. After $30 \mathrm{~s}$ first the helium flow was switched off followed by switching off the voltage. The total analysis time was approximately $45 \mathrm{~s}$.

\section{Coated Blade Spray-MS Measurements}

For CBS analysis, both the ion sources of the Orbitrap and the triple-quadrupole MS were slightly modified. Again, for the Orbitrap, the ion source was opened, and the security lock was overruled. The ion source of the Quattro Ultima was modified by removing the ESI probe and the glass housing; the security lock was overruled by placing a pin in the interlock. To create a high voltage on the blade an external power supply was used. The positive electrode was connected to the blade and the negative electrode was connected to the ground of the mass spectrometer. The voltage on the blade was set at $3.5 \mathrm{kV}$, and spectra were recorded for $30 \mathrm{~s}$. Comparable settings as described in the DAPCI part were used for the Orbitrap experiments. Also, data collection and background subtraction were performed as previously indicated using Masslynx (version 4.1). For the triple-quadrupole tandem MS analysis, the following settings were used: positive electrospray ionization (ESI) mode, desolvation temperature of $300^{\circ} \mathrm{C}$, source temperature of $120^{\circ} \mathrm{C}$, and a cone voltage of $35 \mathrm{~V}$. The desolvation gas was nitrogen and the collisioninduced dissociation (CID) gas was argon; the dwell time was set to $100 \mathrm{~ms}$.

To prepare the coated blades for analysis they were preconditioned with $\mathrm{MeOH}$ :water $(1: 1 \mathrm{v} / \mathrm{v} \%)$ solution and vortexed for $2 \mathrm{~min}$ at $2500 \mathrm{rpm}$. CBS-MS analyses was carried out following two different procedures: (1) $20 \mu \mathrm{L}$ of sample is directly transferred to the blade, or (2) the coated blade is first placed in a vial containing $1 \mathrm{~mL}$ of the sample extract and mixed using a vortex for $1 \mathrm{~min}$. For both procedures, the coated blade was rinsed using water and vortexed at $2500 \mathrm{rpm}$ for $10 \mathrm{~s}$. Desorption and CBS ionization were carried out using a drop of $20 \mu \mathrm{L}$ of $\mathrm{MeOH}$ containing $0.1 \mathrm{v} / \mathrm{v} \%$ formic acid. After, and between analysis, blades were cleaned for re-use using MeOH:ACN:IPA (50:25:25 v/v \%) solution and vortexed for $1 \mathrm{~min}$ at $2500 \mathrm{rpm}$. For calf urine experiments, the compounds of interest were spiked into blank urine in a concentration range of 1 to $100 \mu \mathrm{g} / \mathrm{L}$. The coated blades were used according to a previously described procedure (Gómez-Ríos and Pawliszyn 2014; Gómez-Ríos et al. $2017 \mathrm{a}, \mathrm{b})$ except for the wash of the blade which was now done with $1 \mathrm{ml}$ acetic acid $(1.0 \mathrm{M})$, methanol and acetone: chloroform $(1: 1 \mathrm{v} / \mathrm{v})$ at $2500 \mathrm{rpm}$ for $10 \mathrm{~s}$ each.

\section{Data Processing}

For constructions of the calibration curves, the area under the specific ion signal in the reconstructed ion chromatogram (RIC) was used without any smoothing algorithm. For determination of the limit of detection, the first concentration in the calibration curve with an area higher than the previous lower calibration concentration were used.

\section{Results and Discussion}

For future on-site food analysis by (trans)portable ambient ionization MS, there are several requirements as described in the introduction, most importantly the technique should be robust, preferably self-providing ionization without the need for complicated external supplies and gasses, and easy to operate for non-expert operators. Besides these practical requirements, there are also analytical requirements such as capable of performing repeatable measurements in the field. Furthermore, the techniques should provide fit-for-purpose sensitivity for the selected applications, which should result in a low number of false-negative screening results. Each technique explored was assessed according to these requirements. Experimental parameters such as temperature, gas flows, distance, and angle to MS inlet were first optimized for each selected ambient ionization technique.

\section{Matrix-Assisted Inlet Ionization}

There are several MAI-related techniques available: matrixassisted inlet ionization (MAII), matrix-assisted ionization vacuum (MAIV), and solvent-assisted ionization (SAII) (Li et al. 2012). Here, the applicability of MAII was explored as it was considered as the most practical for future on-site application. The main advantage of MAII for on-site measurements is its simplicity as gases, power supplies, and/or lasers are not needed. MAII is a technique in which the ionization relies solely on the sublimation of the matrix (e.g., 3-NBN) together with the sample extract in front of the inlet of a MS. In practice, first a sample extract is spotted on a glass slide, next the MAI matrix is added and then the slide is dried. The dried glass slide is gently tapped against the inlet of the MS aiming for a rapid sublimation of the matrix and the analyte; see the schematic overview in Fig. 1a.

An obvious robustness concern is that following tapping of a sample matrix against the inlet, the MS will get severely 

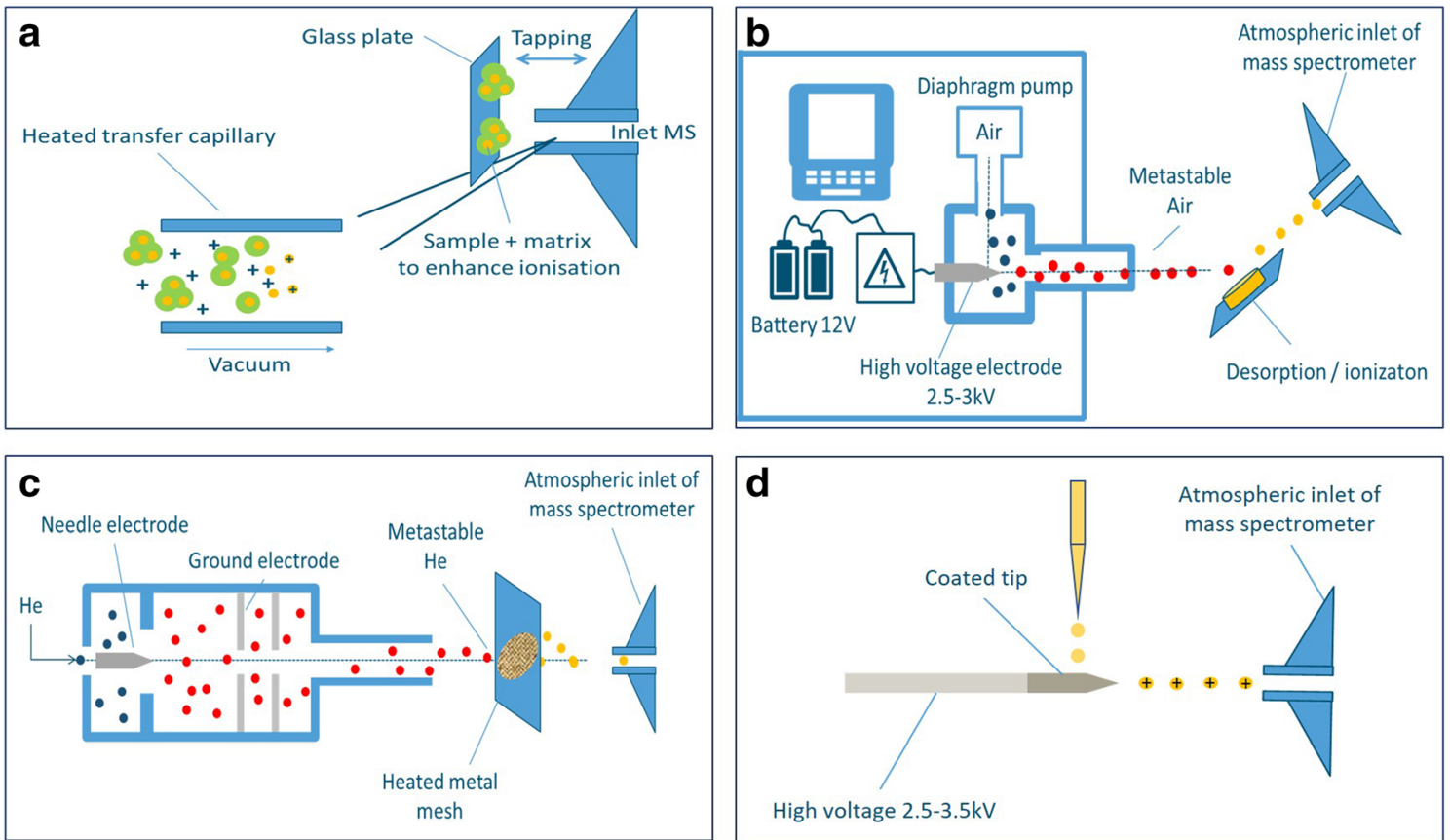

Fig. 1 a Schematic overview of MAII. b Schematic overview of handheld DAPCI. c Schematic overview of simplified TM-DART. d Schematic overview of coated blade spray MS

contaminated and even clogging of the MS inlet may be expected. In practice, however, we did not encounter problems with clogging although we did observe a gradual increase in background signal. As a result, cross-contamination cannot be ruled out thus causing false-positive results in food control.

Furthermore, the stability of MAI sample preparation was considered since differences in time between preparing the sample-matrix and the actual (sequential) MS analysis will occur. Therefore, the MAI matrix was fortified with levofloxacin and clenbuterol on two different glass slides. To one of the spots, 3-NBN was added directly and dried, and to the other spot, 3-NBN was added after $17 \mathrm{~h}$. Within those $17 \mathrm{~h}$, the ion abundance of the various veterinary drugs decreased by approximately $40 \%$ as compared to a sample with freshly added MAI matrix. In practice, this will not lead to many problems as long as the time between MAI preparation and MS analysis remains much shorter.

For MAII, the sensitivity was determined by analyzing standards from 0.1 to $50,000 \mu \mathrm{g} / \mathrm{L}$. Both levofloxacin and dapsone were detected at all concentrations tested, clenbuterol from $10 \mu \mathrm{g} / \mathrm{L}$ and domoic acid from $100 \mu \mathrm{g} / \mathrm{L}$. Concerning precision, the fluctuation in signal intensity was huge and no calibration curve could be constructed reliably. The repeatability was determined by analyzing ten different sample spots of the various test compounds at the same concentration. In general, isotopically labeled internal standards are used to compensate for losses during sample clean-up or to correct for fluctuations in the signal intensity. Unfortunately, isotope standards are not available for many of the food contaminant standards tested. All ten measurements showed a signal, so no false-negative results were obtained at a level of $1000 \mu \mathrm{g} / \mathrm{L}$ in standard solutions: CVs ranged from $45 \%$ for dapsone to $126 \%$ for clenbuterol (Table 1). For practical use, detection levels should be much lower; for example, the recommended concentration for analysis of clenbuterol is $0.2 \mu \mathrm{g} / \mathrm{L}$ in urine, which is much lower than the $10 \mu \mathrm{g} / \mathrm{L}$ standard solution as used in this study. For other compounds, MAII could be potentially sensitive enough. Levofloxacin and dapsone are not allowed to be used, so detection levels should be as low as possible. Based on the experiments with standard solutions, the sensitivity is acceptable; however, due to the poor reproducibility, MAII would cause too many false negatives. Experimental parameters, such as the force of tapping and tapping of a so-called hotspot, are not easily manually controlled. An improvement for this technique could be the development of a "MAI-tapper" that assures a constant tapping force. But MAII is inherently by design not very reproducible, although appealing for its great simplicity an initial proof of concept MAII was explored in the analysis of blueberries fortified with chlormequat. After adding 3-NBN matrix on the peal of the blueberry, the blueberry itself was tapped directly to the MS inlet. A MAI MS spectrum was obtained with the expected $\mathrm{m} / \mathrm{z}$ and chlorine isotopic distribution of chlormequat (Fig. 2a). Fair to say that chlormequat already has a permanent charge so the desorption is more important than the actual ionization here, but it highlights that MAII may be considered for food contaminant analysis even directly from a food surface. This is in line with literature (Devereaux 2016) in which another permanently charged pesticide, the dication paraquat, was successfully measured at $10 \mathrm{ng}$ on a tulip leaf by MAI. 
From this evaluation of MAII, it can be concluded that MAII is a simple ionization technique with some potential to be used on-site. However, for realistic food safety applications, the reproducibility is rather poor. Although others did not find any evidence of sample carry-over (Devereaux 2016), there is an inherent high risk of cross-contamination, potentially yielding high numbers of false-positive screening results. Overall, these drawbacks outweigh the benefits of the technique and MAII is not considered to be directly applicable for on-site measurements in food safety soon.

\section{Handheld DAPCI Ionization}

The second technique explored was a handheld, batterypowered desorption atmospheric pressure chemical ionization (DAPCI) device. DAPCI uses a high DC voltage $(3 \mathrm{kV})$ on a needle to generate a corona discharge and thereby gas-phase reagent ions are formed from ambient air and pneumatically transferred via a nozzle onto the surface of the sample. On the surface, the analytes are desorbed and chemically ionized and transported towards the inlet of the MS due to the electrostatic field and some vacuum drag. The handheld DAPCI device can be battery operated for $1 \mathrm{~h}$, making it ideal for on-site analysis. Figure $1 \mathrm{~b}$ gives a schematic overview of the DAPCI device.

Initial experiments with the DAPCI device were performed on an Orbitrap mass analyzer, followed by experiments on the transportable single-quadrupole MS. The DAPCI Orbitrap experiments demonstrated the successful ionization of all compounds containing secondary amines. Unfortunately, other compounds having less proton affinity and/or unfavorable desorption characteristics were not ionized at all. DAPCI was not very sensitive: typical detection starts at concentrations of $100 \mu \mathrm{g} / \mathrm{mL}$ from which $20 \mu \mathrm{L}$ is applied on the slide. In practice, this would mean that detection limits will be in the $\mathrm{ppm}(\mathrm{mg} / \mathrm{L}-\mathrm{mg} / \mathrm{kg})$ range which is rather high for regulatory food control and will be above most of the currently applied maximum residue limits. Optimization parameters such as the reflection angle, distance to MS inlet, or the analysis time did not yield an improved response. In contrast, the surface material where the sample is spotted on did show an effect: a glass slide yielded a higher signal than other types of surfaces such as filter paper, PTFE slides, or PEEK slides. Most likely this is due to incomplete desorption due to the absorption of the compounds of interest onto the hydrophobic target materials. Direct analysis of spiked food samples was performed; for example, $5 \mu \mathrm{L}$ of $100 \mu \mathrm{g} / \mathrm{mL}$ atrazine was spiked onto the surface of a pumpkin (Fig. 2b). Next, a slice of pumpkin peal was positioned close to the inlet of the MS and the DAPCI device was simply directed onto the food sample. These measurements were successful; however, sensitivity was poor and optimal positioning of the handheld DAPCI in front of the MS is challenging. Following these initial Orbitrap experiments, additional experiments were performed on the transportable 

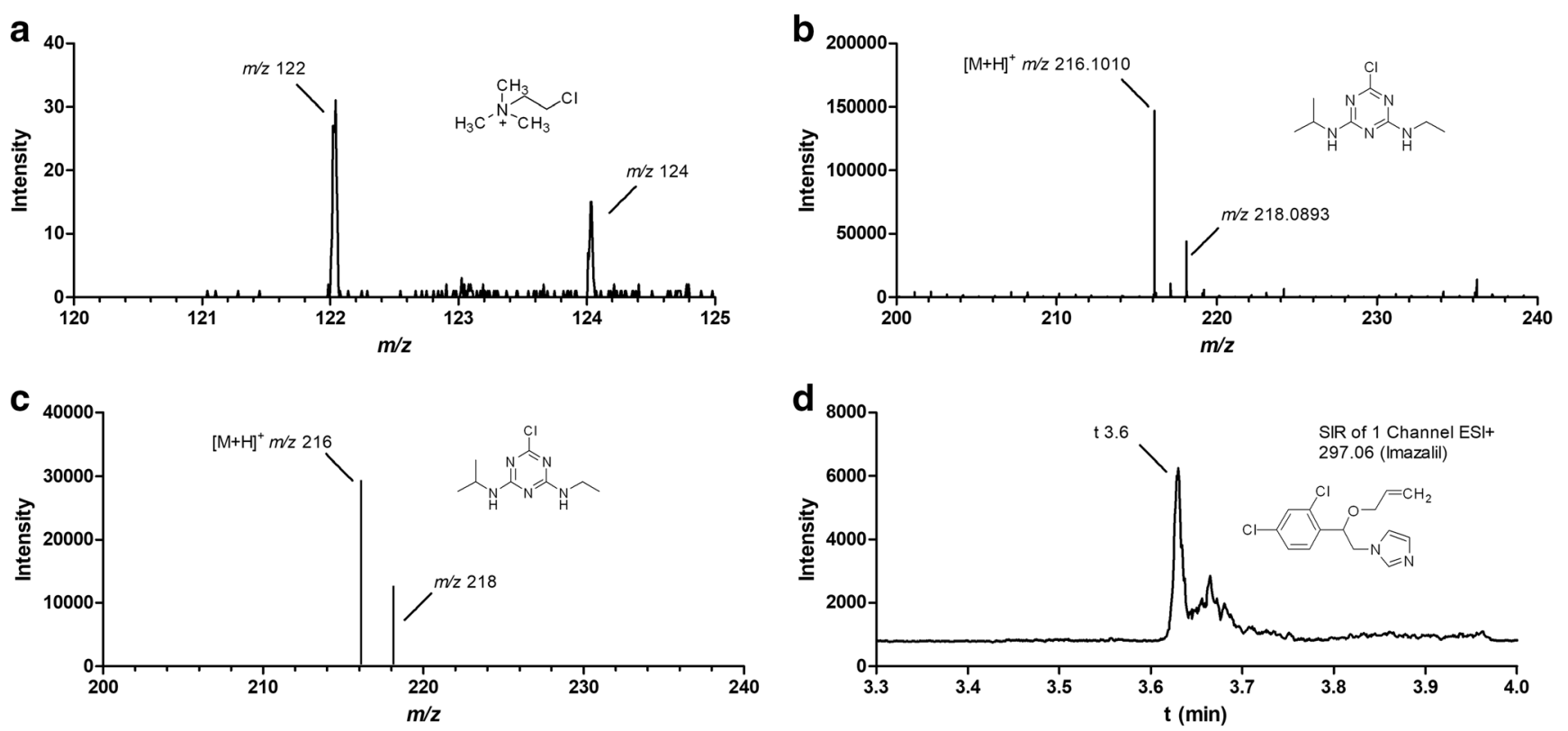

Fig. 2 a Mass spectrum obtained from MAII analysis of chloromequat spiked on a blueberry. b High-resolution accurate mass spectrum obtained by reflection-mode handheld DAPCI-Orbitrap analysis of atrazine spiked on a pumpkin. c Low-resolution mass spectrum obtained from transmission-mode handheld DAPCI analysis on a transportable single-

single-quadrupole MS. The design of the QDa source, due to space limitations, did not allow the use of the DAPCI source in reflection mode. Instead, transmission mode DAPCI was tried by placing a glass tube directly in between the DAPCI beam and the sample cone of the MS. The sensitivity of the DAPCI was determined by analyzing approximately $5 \mu \mathrm{L}$ of standard solutions ranging from 0.1 to $2000 \mu \mathrm{g} / \mathrm{mL}$. On this transportable single-quadrupole MS instrument, a signal could be observed for atrazine standard solutions from $100 \mu \mathrm{g} / \mathrm{mL}$ onwards. The repeatability was determined by analyzing ten times a standard of atrazine (Table 1). Unfortunately, from the 10 repeated analysis in three cases, no atrazine was detected at all. In a subsequent experiment, an apple spiked with a few $\mu \mathrm{L}$ of a $100 \mu \mathrm{g} / \mathrm{mL}$ standard solution was wiped with a Dip-It sampler and analyzed by using the handheld DAPCI in transmission mode. The mass spectrum obtained shows the $\mathrm{m} / \mathrm{z}$ and chlorine isotope distribution of atrazine (Fig. 2c). In literature hardly any handheld DAPCI data can be found for comparison (Jjunju et al. 2015): the technique has been combined with an ion-trap MS in negative ion full scan MS and $\mathrm{MS}^{\mathrm{n}}$ modes and the authors reported an absolute sensitivity for standards of nitroaromatic explosives spotted onto filter paper of $6 \mathrm{pg}$ absolute and a repeatability of $7 \%$ rel.SD $(n=3)$ at $10 \mathrm{ng} / \mathrm{mL}$. In sharp contrast, our device in positive ion single-stage MS mode showed a detection limit in the order of a few hundred ng and repeatability of $30-50 \%(n=10)$. However, it should be noted that the handheld DAPCI is not yet a commercial produced device with associated QA/QC. So apart from the obvious application differences, at least one other explanation

quadrupole MS of atrazine spiked on an apple. d Reconstructed ion chronogram obtained from the analysis of imazalil in a tequila drink following transfer of the fungicide from a lemon slice into the tequila, using the setup of Fig. 1c

would be that our handheld DAPCI is just a second prototype, manufactured by the inventors upon our request.

From our initial evaluation of handheld DAPCI, it can be concluded that this prototype device is truly handheld and, at least in theory, an ideal device for on-site measurements due to the absence of an external high voltage and heavy gas supply and its battery operation. However, at the current development stage, the repeatability and sensitivity are still poor which will yield an unacceptably high percentage of false-negative onsite screening results for regulated substances.

\section{Simplified Transmission-Mode DART Ionization}

A simplified transmission-mode DART (TM-DART) setup was evaluated, which is a slightly modified version of the original but discontinued ID-CUBE device. The ID-CUBE housing was removed to allow a closer positioning at the MS inlet. An external power source was used to control the discharge electrode. Compared to a commercial DART setup, this setup does not have a gas heater, grid electrode, nor a VAPUR interface. In our TM-DART, the sample is spotted onto a credit card like sampling card having a metal screen. For analysis, the card is simply inserted into a slot where two electrodes provide resistive heating to support thermal desorption of the sample from the grid. The power supply has 3 settings, i.e., low, medium, high, which correlates respectively with approximately 200,350 , and $450{ }^{\circ} \mathrm{C}$. Ionization of the desorbed analytes occurs via metastable helium and subsequent ion-molecule reactions with the ambient environment. 
For on-site applications, a small helium cylinder would be required which is a compromise versus our (trans)portability requirements. Figure $1 \mathrm{c}$ shows a schematic overview of the TM-DART principle. For each experiment, the helium flow rate, temperature setting, and distance between the TM-DART and MS inlet were optimized. Standard solutions obtained from an inter-laboratory study on ambient ionization organized by the British Mass Spectrometry Society (BMSS) were used. The study consisted of a sample containing seven compounds and a single internal standard. The study of the BMSS aimed at the repeatability and reproducibility of various ambient ionization techniques. The performance of our simplified TM-DART was critically compared with the commercial DART, both using the same high-resolution benchtop Orbitrap MS instrument.

For the compounds with a low molecular weight (paracetamol ion at $\mathrm{m} / \mathrm{z}, 152$ and ibuprofen at $\mathrm{m} / \mathrm{z} 207$ ), the signal height using the commercial DART device was somewhat higher compared to the simplified TM-DART setup. In contrast, the TM-DART performed better for the compounds with a higher molecular weight (diclofenac at $m / z 297$, colchicine $m / z, 400$ and terfenadine $m / z 472$ ), see also Fig. 3 .

A probable explanation for the increased signal in TMDART at higher masses is the direct resistive heating of the sample grid, while in the commercial DART-SVP device, the actual temperature measured on the sample is much lower than the temperature of the gas heater. Furthermore, when using a Dip-It sampler in conventional DART, a small part of the sample volume may remain on the tip while in TMDART, $5 \mu \mathrm{L}$ is pipetted onto the metal grid of the OpenSpot sample card and directly heated. Both DART systems achieved similar repeatability for the different analytes with paracetamol and ibuprofen being an exception, see Table 1 . The repeatability errors of paracetamol and paracetamol-d4 were somewhat higher with the TM-DART, respectively $37.4 \%$ and $37.7 \%$, than with the DART-SVP, respectively $24.7 \%$ and $31.4 \%$. The same analytes were tested using the TM-DART connected to the (trans)portable single-quadrupole MS. Similar to the Orbitrap MS experiments, terfenadine showed the most intense average signal. All other compounds were detected as well, demonstrating the possibility to couple the TM-DART to a simple transportable low-resolution MS. Next, the sensitivity of the TM-DART single-quadrupole MS system was assessed in triplicate using a mixture containing atrazine, clenbuterol, metolachlor, stanozolol, cortisol, and tetracycline $(5 \mu \mathrm{L}$ of a $1000,500,100,50,10,5$, and $1 \mu \mathrm{g} / \mathrm{L}$ solution). Tetracycline yielded false-negative results, most probably caused by binding of the tetracycline to the metal grid surface of the OpenSpot cards. However, all other compounds could be detected at concentrations as low as $1 \mu \mathrm{g} / \mathrm{L}$, which is very promising for real-life applications in food contaminant analysis since these concentration levels in standards are in practice close to the recommended concentration limits of $0.2-2 \mu \mathrm{g} / \mathrm{L}$ for clenbuterol, stanozolol, and cortisol in urine and below the maximum residue limits of $10-50 \mu \mathrm{g} / \mathrm{kg}$ for atrazine, metolachlor, and tetracycline in food products. To demonstrate the potential for food analysis, a lemon sample was transferred to the metal grid of the sample card by simply scratching of the lemon peel. Subsequently, the sample card was placed in the TM-DART source and analyzed with the (trans)portable single-quadrupole MS system. The extracted ion chronogram showed a clear peak for the ion $\mathrm{m} / z$ 298, including a characteristic chlorine isotopic pattern, which could be related to imazalil which is typically used as a fungicide in lemon cultivation. Washing the lemon peel with methanol significantly decreased the imazalil concentration $(>70 \%)$. Therefore, it can be hypothesized that if lemon slices are used in an alcoholic beverage, then the imazalil could easily transfer from the lemon to the drink. This was confirmed by placing a piece of lemon in a glass of tequila for $10 \mathrm{~min}$, followed by the transfer of a $5 \mu \mathrm{L}$ tequila aliquot to the metal grid of the sample card. As a result, imazalil residues were rapidly and easily detected in the tequila samples tested (Fig. 2d). In general, the results are consistent with the DART applications described in literature (Hajslova et al. 2011; Farré et al. 2013): without internal standard, repeatability is poor but this can be improved in many cases by the application of an appropriate internal standard, allowing screening at low $\mu \mathrm{g} / \mathrm{L}$ levels.

Overall, it can be concluded that TM-DART single-quadrupole (trans)portable MS is a robust and easy to use technology compared to MAII and handheld DAPCI. The technique is capable of detecting a broad range of relevant food contaminants in a sensitive and reproducible manner. The biggest drawback and compromise are the need for a helium gas supply and an external high voltage power supply (when not adequately supplied by the transportable MS analyzer).

\section{Coated Blade Spray-MS}

Coated blade spray (CBS) combines solid-phase microextraction (SPME) with direct desorption/ionization under ambient MS conditions; for a schematic overview, see Fig. $1 \mathrm{~d}$. The analysis by CBS consists of multiple steps that include preconditioning of the sorbent and immersion into a vial containing the sample extract. The extraction proceeds under vortex conditions, followed by a quick washing step to remove some matrix components that may cause ion suppression. Subsequently, the metal blade is simply positioned in front of the MS inlet. Following the application of a drop of a desorption solution, a high voltage is applied to the blade, and electrospray ionization of the analytes occurs at the tip of the blade. Although some preparation steps are needed for CBS, they still could be easily performed on-site. Importantly, no gas supply is needed, and the power supply of the MS can be used to apply the high voltage directly onto 
Fig. 3 Comparison of the performance of TM-DART vs SVP-DART for different compounds $(n=6)$
TM- vs SVP-DART Exactive measurements

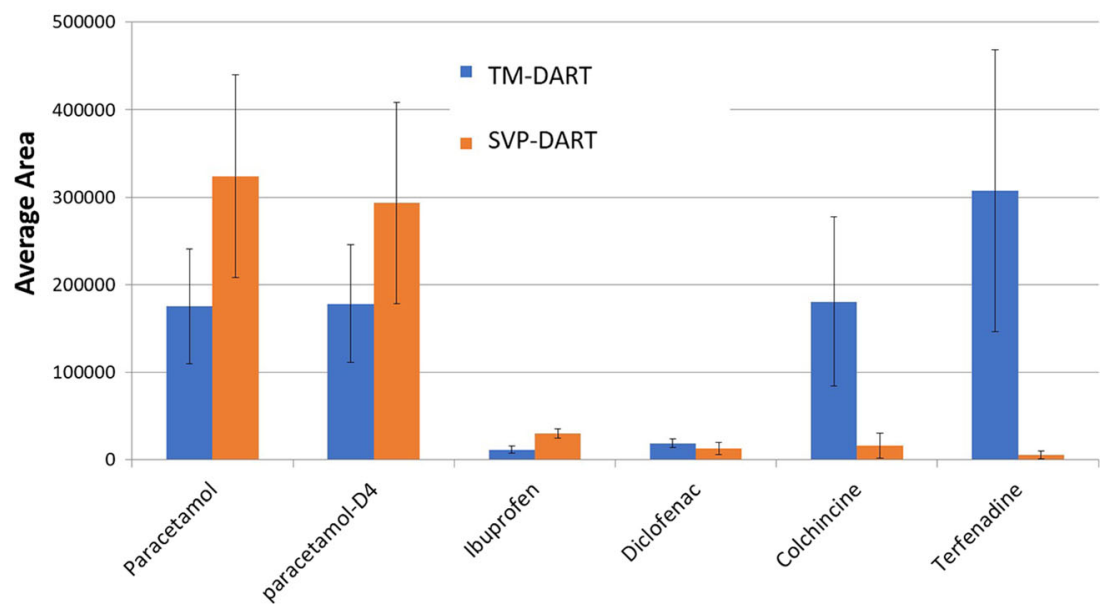

the CBS blade. Furthermore, based on the stationary phase chemistry on the blades, additional selectivity can be expected that allows both a reduction of any matrix effect and simultaneous trace enrichment of the compounds of interest.

The sensitivity of the CBS-MS was determined by recording calibration curves for a mixture of beta-agonists in the concentration range of 1 to $100 \mu \mathrm{g} / \mathrm{L}$. The analysis was performed on a benchtop high-resolution Orbitrap MS. For all beta-agonists tested, similar results were obtained demonstrating good linearity $\left(R^{2}>0.99\right)$ and sensitivity $(1 \mu \mathrm{g} / \mathrm{L})$ in solvent standards. Compared to the other techniques tested, CBS provides the most sensitive and linear results. The repeatability was determined by fortifying blank calf urine at 1 and $5 \mu \mathrm{g} / \mathrm{L}$ with a mixture of beta-agonists. Since the whole blade is immersed in the urine samples, the extraction will occur at both sides of the blade while desorption and ionization is performed at one side at a time. This allows performing a duplicate measurement using the other side of the blade. In all samples tested, it was possible to detect clenbuterol with a repeatability of $30.2 \%$. For semi-quantitative on-site screening of veterinary drug residues, these data are more than adequate and will further improve using stable isotope-labeled internal standards. The recommended concentration for beta-agonists in urine is, depending on the compound, between 0.2 and $10 \mu \mathrm{g} / \mathrm{L}$; the obtained results suggest that detection of beta-agonists in urine is possible at the relevant concentrations. These findings are in line with early results in literature (Gómez-Ríos and Pawliszyn 2014; Gómez-Ríos et al. 2017a, b) in which low- and sub- $\mu \mathrm{g} / \mathrm{L}$ (in MS/MS) detectability was reported for several drugs (including cocaine, diazepam, salbutamol, clenbuterol, and stanozolol) and excellent repeatability, provided an isotope-labeled internal standard was used.

When CBS is used in combination with a low-resolution single-quadrupole mass spectrometer, the detection of the specific $m / z$ of clenbuterol will be more challenging. To mimic such a situation, the Orbitrap data from spiked urine were further examined, see Fig. 4a. Already at mass resolution, 25,000 (full-width half-maximum at $\mathrm{m} / \mathrm{z}, 200$ ) interfering ions are present next to the $m / z$ of clenbuterol and its internal standard. It was demonstrated (Berendsen et al. 2013) that predictable selectivity can be obtained using triple-quadrupole MS measurements, even without liquid chromatographic separation. Currently, there is no (trans)portable triplequadrupole MS on the market, so to mimic the performance of a future (trans)portable low-resolution triple-quadrupole MS, a 15-year-old triple-quadrupole mass analyzer was used to perform CBS MS/MS measurements. In Fig. 4b, an example is given of the measurement of blank urine and urine spiked at $1 \mu \mathrm{g} / \mathrm{L}$ for different beta-agonists on this CBS-MS/MS setup. For all compounds, the spike could be detected at $1 \mu \mathrm{g} / \mathrm{L}$ in a real-life sample matrix using the selected specific ion transitions. This demonstrates the power of triple-quadrupole measurements in a complex matrix with minimal clean-up and direct measurement of drugs at trace levels following coated blade spray MS.

\section{Conclusions}

Based on the requirements for ambient ionization for future food analysis using (trans)portable mass spectrometry the following can be concluded. From the techniques tested, MAII and DAPCI fulfilled the simplicity criteria but the analytical performance of both techniques was rather poor. Both techniques lacked the sensitivity to detect compounds of interest at recommended concentrations and robustness needed for reliable on-site food analysis. The performance greatly improved with the use of the simplified TM-DART and CBS ambient ionization approaches. TM-DART was easy to operate, sensitive enough to detect compounds at recommended concentrations and provided (relatively) reproducible results, but a main drawback for future on-site analysis remains the requirement 

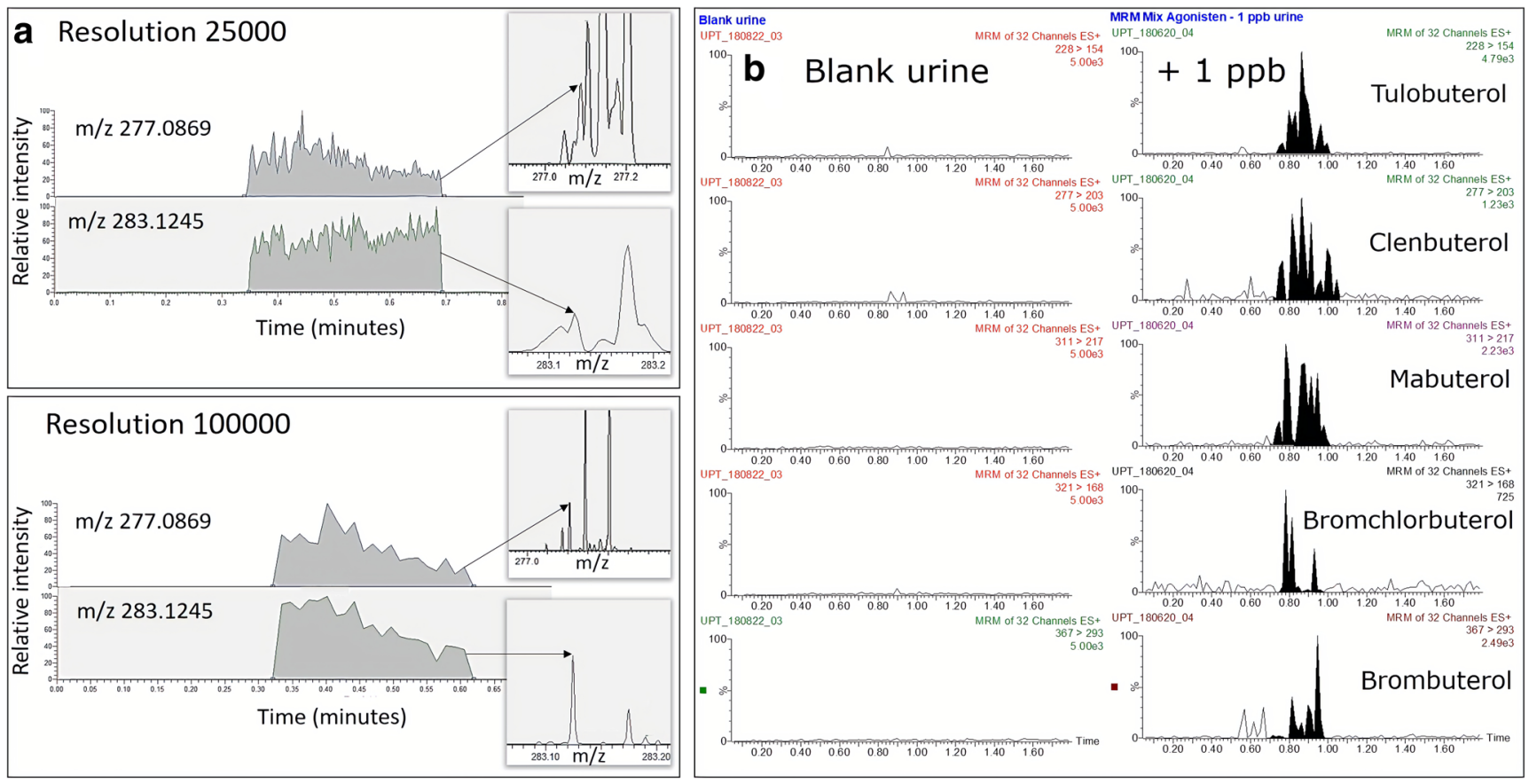

Fig. 4 a CBS-Orbitrap HRMS analysis of a spike of $1 \mu \mathrm{g} / \mathrm{L}$ clenbuterol to calve urine at mass resolution 25,000 and 100,000. The insets show the average spectrum of clenbuterol and clenbuterol-D6. b CBS-triple-

of a helium gas supply and in some cases an external high voltage supply. CBS could be operated without the use of gas and external power supply and, following this initial evaluation, is considered highly promising for future on-site analysis of liquid samples or extracts. Detection of beta-agonists at recommended concentrations in urine was possible with CBS mass spectrometry.

When applying these ionization techniques without sample preparation and/or chromatographic separation prior to detection using a single-stage low- or high-resolution mass analyzer, it is clear that the lack of overall selectivity must be compensated to allow on-site screening of regulated substances at relevant levels. First of all, the (trans)portable MS should provide more selectivity which points into the direction of tandem MS, either based on ion-trap or triple-quadrupole mass analyzers. Besides, some selectivity during sample introduction might be highly beneficial as, for example, employing (selective) stationary phase materials coated onto TM-DART grids and CBS blades. Without these, the number of falsepositive and false-negative screening results in food contaminant analysis at regulatory limits will be most likely too large.

Acknowledgments The presented work was performed by a group of enthusiast students doing their internship at the RIKILT institute (nowadays Wageningen Food Safety Research): we would like to thank Jorien Hattink, Hua Easton, Josha Jager, Michael van Dam, and Rachelle Linders for their contributions. KR Analytical is thanked for the gift of ID-Cube parts and OpenSpot cards, Waters for the gift of a QDa ion source block. Dr. German Gomez-Rios nd Dr. Varoon Singh from the University of Waterloo is acknowledged for the production of coated blades. quadrupole MS/MS of a blank calve urine and a spike of different betaagonists at the $1 \mu \mathrm{g} / \mathrm{L}$ trace level

Funding Information This project was financially supported by the Dutch Ministry of Agriculture, Nature and Food Quality (project KB23-002-019).

\section{Compliance with Ethical Standards}

Conflict of Interest The authors declare that they have no conflict of interest.

Human and Animal Rights Not applicable.

Informed Consent Not applicable.

Open Access This article is licensed under a Creative Commons Attribution 4.0 International License, which permits use, sharing, adaptation, distribution and reproduction in any medium or format, as long as you give appropriate credit to the original author(s) and the source, provide a link to the Creative Commons licence, and indicate if changes were made. The images or other third party material in this article are included in the article's Creative Commons licence, unless indicated otherwise in a credit line to the material. If material is not included in the article's Creative Commons licence and your intended use is not permitted by statutory regulation or exceeds the permitted use, you will need to obtain permission directly from the copyright holder. To view a copy of this licence, visit http://creativecommons.org/licenses/by/4.0/.

\section{References}

Berendsen BJA, Stolker LAM, Nielen MWF (2013) The (un)certainty of selectivity in liquid chromatography tandem mass spectrometry. J Am Soc Mass Spectrom 24:154-163 
Berendsen BJA, Wegh RS, Meijer T, Nielen MWF (2015) The assessment of selectivity in different quadrupole-orbitrap mass spectrometry acquisition modes. J Am Soc Mass Spectrom 26:337-346

Brkic B, France N, Giannoukos S, Taylor S (2018) An optimised quadrupole mass spectrometer with a dual filter analyser for in-field chemical sniffing of volatile organic compounds. Analyst 143: 3722-3728

Bu X, Regalado EL, Hamilton SE, Welch CJ (2016) The emergence of low-cost compact mass spectrometry detectors for chromatographic analysis. Trends Anal Chem 82:22-34

Chen H, Zheng J, Zhang X, Luo M, Wang Z, Qiao X (2007) Surface desorption atmospheric pressure chemical ionization mass spectrometry for direct ambient sample analysis without toxic chemical contamination. J Mass Spectrom 42:1045-1056

Cody RB, Laramee JA, Durst HD (2005) Versatile new ion source for the analysis of materials in open air under ambient conditions. Anal Chem 77:2297-2302

Cooks RG, Ouyang Z, Takats Z, Wiseman JM (2006) Ambient mass spectrometry. Science 311:1566-1570

Devereaux ZJ, Reynolds CA, Fischer JL, Foley CD, DeLeeuw JL, Wager-Miller J, Narayan SB, Mackie K, Trimpin S (2016) Matrixassisted ionization on a portable mass spectrometer: analysis directly from biological and synthetic materials. Anal Chem 88:1083110836

Farré M, Pico Y, Barceló D (2013) Direct peel monitoring of xenobiotics in fruit by direct analysis in real time coupled to a linear quadrupole ion trap - orbitrap mass spectrometer. Anal Chem 85:2638-2644

Feider CL, Krieger A, DeHoog RJ, Eberlin LS (2019) Ambient ionization mass spectrometry: recent developments and applications. Anal Chem 91:4266-4290

Garcia-Reyes JF, Jackson AU, Molina-Diaz A, Cooks RG (2009) Desorption electrospray ionization mass spectrometry for trace analysis of agrochemicals in food. Anal Chem 81:820-829

Gómez-Ríos GA, Pawliszyn J (2014) Development of coated blade spray ionization mass spectrometry for the quantitation of target analytes present in complex matrices. Angew Chem Int Ed 53:14503-14507

Gómez-Ríos GA, Tascon M, Reyes-Garcés N, Boyac1 E, Poole J, Pawliszyn J (2017a) Quantitative analysis of biofluid spots by coated blade spray mass spectrometry, a new approach to rapid screening. Sci Rep 7:16104

Gómez-Ríos GA, Vasiljevic T, Gionfriddo E, Yu M, Pawliszyn J (2017b) Towards on-site analysis of complex matrices by solid-phase microextraction transmission mode coupled to a portable mass spectrometer via direct analysis in real time. Analyst 142:2928-2935

Hajslova J, Cajka T, Vaclavik L (2011) Challenging applications offered by direct analysis in real time (DART) in food-quality and safety analysis. Trends Anal Chem 30:204-218

Harding LP, Parkes GMB, Townend JD (2014) Parameters affecting ion intensities in transmission-mode direct analysis in real-time mass spectrometry. Analyst 139:4176-4180

Harper JD, Charipar NA, Mulligan CC, Zhang XR, Cooks RG, Ouyang Z (2008) Low temperature plasma probe for ambient desorption ionization. Anal Chem 80:9097-9104

Harris GA, Galhena AS, Fernandez FM (2011) Ambient sampling/ ionization mass spectrometry: applications and current trends. Anal Chem 83:4508-4538

Harris WA, Henderson DB, Ho JK, Dickinson DN (2015) Comparison of air and nitrogen gas sources for DART mass spectrometry. Poster WP434 presented at the $63^{\text {rd }}$ ASMS Conference on Mass Spectrometry, St. Louis, MO, USA

Huang GM, Xu W, Visbal-Onufrak MA, Ouyang Z, Cooks RG (2010) Direct analysis of melamine in complex matrices using a handheld mass spectrometer. Analyst 135:705-711

Jjunju FPM, Maher S, Li A, Syed SU, Smith B, Heeren RMA, Taylor S, Cooks RG (2015) Hand-held portable desorption atmospheric pressure chemical ionization ion source for in situ analysis of nitroaromatic explosives. Anal Chem 87:10047-10055

Li J, Inutan ED, Wang B, Lietz CB, Green DR, Manly CD, Richards AL, Marshall DD, Lingenfelter S, Ren Y, Trimpin S (2012) Matrix assisted ionization: new aromatic and nonaromatic matrix compounds producing multiply charged lipid, peptide, and protein ions in the positive and negative mode observed directly from surfaces. $\mathrm{J}$ Am Soc Mass Spectrom 23(10):1625-1643

Liu J, Wang H, Manicke NE, Lin JM, Cooks RG, Ouyang Z (2010) Development, characterization, and application of paper spray ionization. Anal Chem 82(6):2463-2471

Liu N, Parra HA, Pustjens A, Hettinga K, Mongondry P, van Ruth SM (2018) Evaluation of portable near-infrared spectroscopy for organic milk authentication. Talanta 184:128-135

Lu H, Zhang H, Chingin K, Xiong J, Fang X, Chen H (2018) Ambient mass spectrometry for food science and industry. Trends Anal Chem 107:99-115

Ma Q, Bai H, Li WT, Wang C, Li XS, Cooks RG (2016) Direct identification of prohibited substances in cosmetics and foodstuffs using ambient ionization on a miniature mass spectrometry system. Anal Chim Acta 912:65-73

McEwen CN, McKay RG, Larsen BS (2005) Analysis of solids, liquids, and biological tissues using solids probe introduction at atmospheric pressure on commercial LC/MS instruments. Anal Chem 77(23): 7826-7831

Nemes P, Vertes A (2007) Laser ablation electrospray ionization for atmospheric pressure, in vivo, and imaging mass spectrometry. Anal Chem 79:8098-8106

Nielen MWF, van Beek TA (2014) Macroscopic and microscopic spatially-resolved analysis of food contaminants and constituents using laser-ablation electrospray ionization mass spectrometry imaging. Anal Bioanal Chem 406:6805-6815

Nielen MWF, Hooijerink H, Zomer P, Mol JGJ (2011) Desorption electrospray ionization mass spectrometry in the analysis of chemical food contaminants in food. Trends Anal Chem 30:165-180

Pulliam CJ, Bain RM, Wiley JS, Ouyang Z, Cooks RG (2015) Mass spectrometry in the home and garden. J Am Soc Mass Spectrom 26:224-230

Rateni G, Dario P, Cavallo F (2017) Smartphone-based food diagnostic technologies: a review. Sensors 17(6):1453

Ross G, Bremer MGEG, Nielen MWF (2018) Consumer-friendly food allergen detection: moving towards smartphone-based immunoassays. Anal Bioanal Chem 410:5353-5371

Schäfer KC, Dénes J, Albrecht K, Szaniszló T, Balog J, Skoumal R, Katona M, Tóth M, Balogh L, Takáts Z (2009) In vivo, in situ tissue analysis using rapid evaporative ionization mass spectrometry. Angew Chem Int Ed 48(44):8240-8242

Schurek J, Vaclavik L, Hooijerink H, Lacina O, Poustka J, Sharman M, Caldow M, Nielen MWF, Hajslova J (2008) Control of strobilurin fungicides in wheat using direct analysis in real time accurate timeof-flight and desorption electrospray ionization linear ion trap mass spectrometry. Anal Chem 80:9567-9575

Smoluch M, Mielczarek P, Silberring J (2016) Plasma-based ambient ionization mass spectrometery in bioanalytical sciences. Mass Spectrom Rev 35:22-34

Snyder DT, Pulliam CJ, Ouyang Z, Cooks RG (2016) Miniature and fieldable mass spectrometers: recent advances. Anal Chem 88:2-29

Soparawalla S, Tadjimukhamedov FK, Wiley JS, Ouyang Z, Cooks RG (2011) In situ analysis of agrochemical residues on fruit using ambient ionization on a handheld mass spectrometer. Analyst 136: 4392-4396

Souza-Silva EA, Gionfriddo E, Pawliszyn J (2015) A critical review of the state of the art of solid-phase microextraction of complex matrices II. Food analysis. Trends Anal Chem 71:236-248 
Takáts Z, Wiseman JM, Gologan B, Cooks RG (2004) Mass spectrometry sampling under ambient conditions with desorption electrospray ionization. Science 306:471-473

Trimpin S (2016) "Magic" ionization mass spectrometry. J Am Soc Mass Spectrom 27:4-21

Trimpin S, Inutan EDJ (2013) Matrix assisted ionization in vacuum, a sensitive and widely applicable ionization method for mass spectrometry. J Am Soc Mass Spectrom 24:722-732

Venter A, Nefliu M, Cooks RG (2008) Ambient desorption ionization mass spectrometry. Trends Anal Chem 27:284-290
Wright S, Malcolm A, Wright C, O'Prey S, Crichton E, Dash N, Moseley RW, Zaczek W, Edwards P, Fussell RJ, Syms RRA (2015) A microelectromechanical systems-enabled miniature triple quadrupole mass spectrometer. Anal Chem 87:3115-3122

Zhai Y, Zhang X, Xu H, Zheng Y, Yuan T, Xu W (2017) Mini mass spectrometer integrated with a miniature ion funnel. Anal Chem $89: 4177-4183$

Publisher's Note Springer Nature remains neutral with regard to jurisdictional claims in published maps and institutional affiliations. 